\title{
ULTRAVIOLET IRRADIATION APPARATUS WITH GRATING MONOCHROMATOR ${ }^{1)}$
}

\author{
TAKASHI ITO \\ Biophysics Laboratory, Department of Physics, Rikkyo \\ (St. Paul's) University, Tokyo, Japan
}

Received June 30, 1964

Attempts to correlate the biological action of ultraviolet light to molecular changes in biological macromolecules have been a main concern among biophysicists and molecular biologists. Especially because of the discoveries of thymine dimer formation by ultraviolet and its interesting reverse reaction by visible light (in coaction with a photoreactivating enzyme), experimental study of molecular mechanisms of mutation induction had, at least, a very convenient tool. However, commercial monochromators do not meet the requirements for such experiments where large doses are necessity.

A prism monochromator with a large aperture may meet the requirements (Fluke and Setlow 1954). However, such an instrument usually needs complicated optical design. In the apparatus reported here, this difficulty is largely circumvented by choosing a large light source of the highest practicable steradiancy. In order to achieve high transmitted power, a relatively large grating was used as a dispersion element in conjunction with a $2-\mathrm{kW}$ xenon arc lamp.

This irradiation appparatus (Figure 1) has been used to provide an "action spectrum" for genetic effect of ultraviolet light in yeast cells in this laboratory (Ito et al. 1964).

Light source: A 2-kW xenon arc lamp (UXL-2000 DV Ushio Kogyo) was chosen for three reasons: (1) this happens to be the largest output lamp available in this country; (2) the arc area is relatively small (high radiant emission); and (3) wavelengths can be selected continuously not only in the ultraviolet region but also in the visible region.

The lamp-house is a steel box $(180 \mathrm{~mm} \times 180 \mathrm{~mm} \times 360 \mathrm{~mm})$ with a quartz window for avoiding unnecessary light and for protection in case of blow-up. Cooling water is run through metal tubing along the wall and a continuous, high-velocity stream of air is also used along the lamp axis in a direct way.

Overall fluctuation of output measured at the exit slit of the monochromator was 1 to $2 \%$ in normal operation.

Monochromator: A grating monochromator with a Czerny-Turner mounting was adopted finally by the recommendation of the technical group of Japan Spectroscopic

1) This work has been supported by a grant from the Toyo Rayon Foundation for the Promotion of Sceince and Technology. 


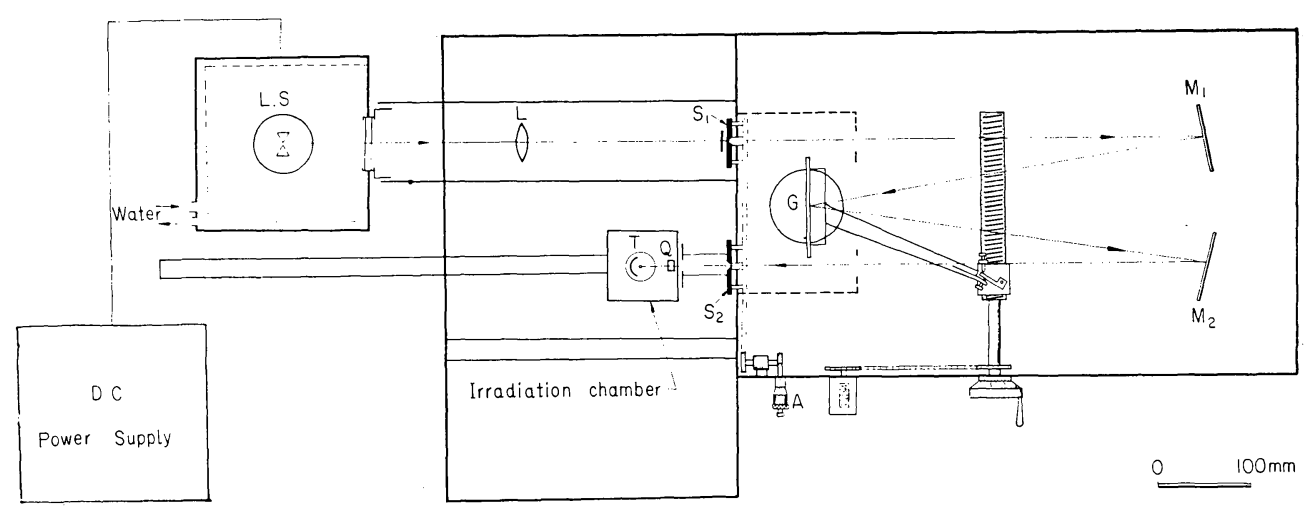

Fig. 1. Ultraviolet irradiation apparatus with grating monochromator.

L. S.: Light source, L: Lens, $\mathrm{S}_{1}$ : Entrance slit, $\mathrm{S}_{2}$ : Exit slit, A: Micrometer,

$\mathrm{M}_{1}$ : Collimator mirror, $\mathrm{M}_{2}$ : Focusing mirror, G: Grating, Q: Quartz cuvette,

T: Photomultiplier tube.

Co. A sketch of the monochromator is shown in Figure 1. A plane reflection grating (purchased from Bausch and Lomb) with 600 grooves $/ \mathrm{mm}(65 \mathrm{~mm} \times 76 \mathrm{~mm}$ ) has been tentatively mounted, although the installation of a 2,160 grooves $/ \mathrm{mm}$ grating had originally been planned. It gives reciprocal dispersion of $33.2 \AA / \mathrm{mm}$ and resolving power of $4.5 \times 10^{4}$ (first order). The various elements, including the irradiation equipment, are supported on an optical bench, although the lamp-house is mechanically disconnected.

The monochromator has been used with the $2-\mathrm{kW}$ xenon lamp as light source placed at a distance of $47.5 \mathrm{~cm}$ from the entrance slit, which is $10 \mathrm{~mm}$ in height and adjustable from 5 to $0 \mathrm{~mm}$ in width with an accuracy of $1 / 100 \mathrm{~mm}$ by a micrometer (both entrance and exit slits being bilaterally adjustable). There is an ample space for putting the various filters between the lamp-house and the entrance slit. Thus, the optical coupling with the light source is not very efficient in view of the optical design of the whole system, but workable intensities have been obtained over the region from $2,300 \AA$ to $3,000 \AA$. An actinometric measurement, made with an alcohol solution of malachite green leucocyanide $14.5 \mathrm{~cm}$ behind the exit slit at the position where the beam spreads out to a useable area of about $17 \mathrm{~mm}$ by $20 \mathrm{~mm}$, gave the intensities shown in Figure 2 for several wavelengths. These intensities were tolerable for mutation induction experiments in yeast cells, although many more intensities are desirable, especially for some in vitro experiments. Future replacement of the grating by the originally designed one $(2,160$ grooves/mm with the same aperture) should give greater intensity and higher spectral precision ${ }^{1)}$.

The level of scattered light was lowered to a negligible amount (less than $3 \%$ ) by putting several slits at the appropriate positions in the monochromator.

1) Recent installation of the new grating $(2,160$ grooves $/ \mathrm{mm})$ has increased the intensities by factors of 4 to 10 depending on the wavelengths in the UV region at even narrower bandwidth of $26 \AA$. 


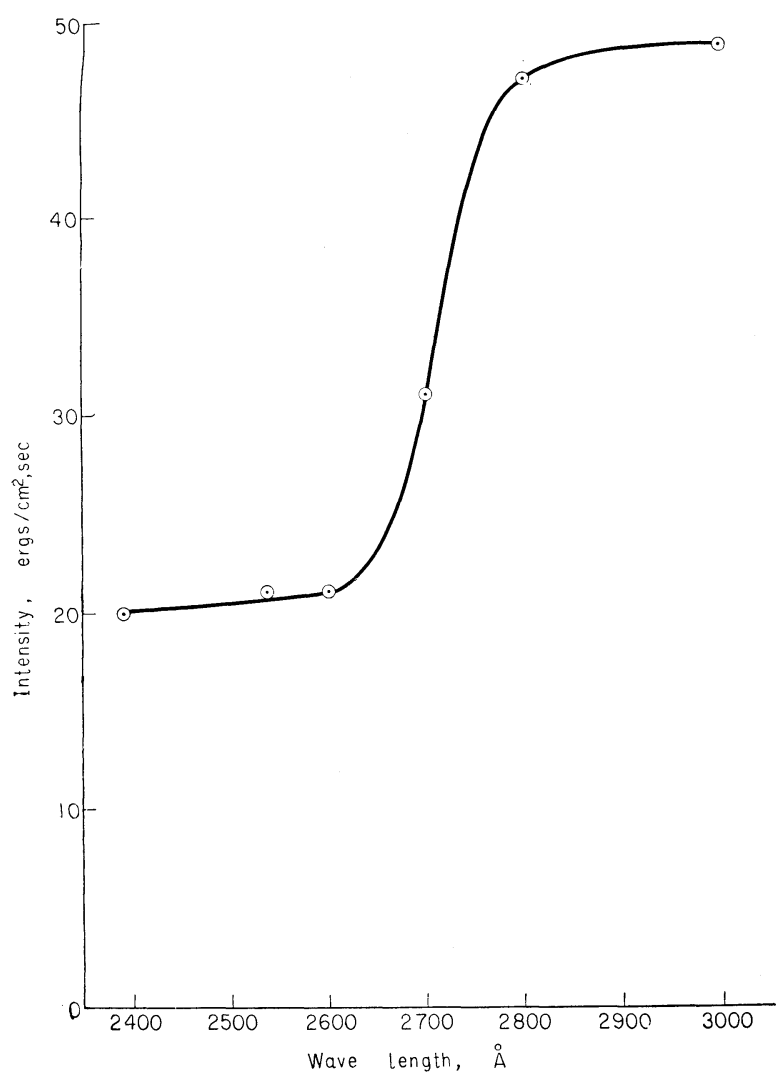

Fig. 2. Intensity of monochromatic ultraviolet light (bandwidth $63 \AA$ ) at a distance of $14.5 \mathrm{~cm}$ from the exit slit.

There is no problem to obtain enough resolving power for the biophysical studies in this type of monochromator. The wavelengths were calibrated with reference to the several lines of cadmium and the counter reads actual wavelengths in $\AA$ unit with an accuracy of $\pm 1 \AA$.

\section{ACKNOWLEDGEMENTS}

The author wishes to express his thanks to the members of the Biophysics Laboratory and to Mr. T. Takenouchi of Japan Spectroscopic Co. for their cooperation during the course of the development of the apparatus. He is also indebted to Professor A. Hagitani and his associates of the Department of Chemistry, Rikkyo University, for the preparation of malachite green leucocyanide. 


\section{LITERATURE CITED}

Fluke, D. J. and R. B. Setlow, 1954 Water-prism ultraviolet monochromators. J. Optic. Soc. Amer. 44: 327-330.

Ito, T., M. Domon, and S. Ishizaka, 1964 Mechanisms of the action of ultraviolet light on biological macromolecule. (in Japanese). Hōshasen Butsuri Kenkyu Suppl. 1: 136-150.

Ito, T., T. Yamasaki, M. Domon, S. Ishizaka and Y. Matsudaira, 1964 Ultraviolet-induced photoreversible genetic change observed in heterozygous diploid system of yeast-A study of the UV action spectrum for induction and of the photoreversion. Jap. J. Genet. 39: 136-146. 